\title{
Application of Stir Tool Force Measuring Dynamometer for Friction Stir Welding of Aluminum Alloys
}

\author{
H. F. Wang, ${ }^{\text {a,bld }}$ J. L. Wang, ${ }^{a}$ D. W. Zuo, ${ }^{b}$ and W. W. Song ${ }^{a}$ \\ ${ }^{a}$ College of Mechanical and Electrical Engineering, Huangshan University, Huangshan, China \\ b College of Mechanical and Electrical Engineering, Nanjing University of Aeronautics and \\ Astronautics, Nanjing, China \\ ${ }^{1}$ wanghnfeng@163.com
}

In this study, a stir tool force measuring dynamometer was designed and manufactured to be suitable for use during the process of friction stir welding. The dynamometer meets the requirements needed for actual force measurements, with error percentage values in the $x-, y-$ and $z$-axis directions of 1.1 , 1.3 , and $1.2 \%$, respectively, and a three-directional sensitivity range of $0.4-2.1 \%$. At the same time, stir tool forces were measured under different process parameters using the manufactured dynamometer. The stir tool force mathematical model, to be used for friction stir welding processes, was established by function approximation and regression analysis methods. The model was set up with a significance level under 90\%. Finally, a comparison between the model-calculated values and experimental values yielded a stir tool force average error of $10.4,4.66$, and $7.11 \%$ in the $x, y$, and $z$ axes direction, respectively. Therefore, calculated and experimental values are in agreement.

Keywords: friction stir welding, dynamometer, octagonal ring, stir tool force model, regression analysis method.

Introduction. Although octagonal ring dynamometers were first used in the field of machine tool cutting, they were later widely adopted in agricultural engineering [1-5]. Some examples of how dynamometers have been used in agricultural engineering include the work of $\mathrm{Xu}$ et al. [6], where an octagonal ring dynamometer was used to measure three-directional wood cutting forces; the work of Karabay [7], where an octagonal ring manufacturing dynamometer was used to measure three-directional drill forces; and the work of Chen et al. [8], where a double extended octagonal ring manufacturing dynamometer was implemented to measure tractor traction. However, to the best of the authors' knowledge, there are no studies, except for [9], on application of a dynamometer to measuring stir tool forces in a friction stir welding process. The stir tool forces exerted during the process of friction stir welding determine the performance of the welding region; therefore, the study of stir tool forces is important. In this study, based on the principles of octagonal ring measuring forces, a dynamometer was designed specifically for stir tool force measurements during friction stir welding. In addition, a stir tool force model was established using the dynamometer. It is expected that the data obtained can be used for subsequent finite element analysis of friction stir welding.

1. Dynamometer Design for Friction Stir Welding. The design of an octagonal ring dynamometer revolves around the ring, and so its working principle is the same as that of a cylindrical ring. However, an octagonal ring is more easily processed than a circular ring, and under the same sensitivity conditions, its stiffness is higher than that of a circular ring.

The octagonal ring working principle is the following: when a horizontal force is applied to the circle, the ring will produce the maximum strain at $45^{\circ}$ to the vertical direction, as shown in Fig. 1a. The strain can be represented as [10]:

$$
\varepsilon_{A}= \pm 313 \frac{F_{x} R}{E b h^{2}},
$$




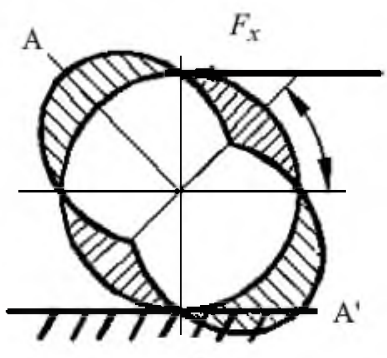

a

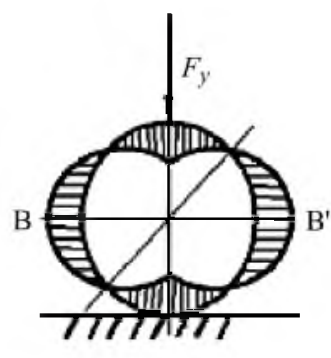

b

Fig. 1. The circular ring force analysis [5].

where $R$ is the average radius of the octagonal ring, $E$ is the elastic modulus, $b$ is the octagonal ring width, and $h$ is the thickness of the octagonal ring.

The horizontal force can be calculated by the deformation that takes place in $A$ and $A^{\prime}$, which is determined using an attached strain gauge.

When a vertical force is applied to the ring, the later will produce the maximum strain along four directions (up, down, left, and right) separated by an interval of $90^{\circ}$, as shown in Fig. 1b. The strain can be represented as [10]:

$$
\varepsilon_{B}=1.09 \frac{F_{x} R}{E b h^{2}} .
$$

Because the up and down directions are not convenient for strain gauge measurements, the strain gauge is placed in the $B$ and $B^{\prime}$ positions.

The octagonal ring body is the elastic element of the dynamometer. The resistance strain gauge is placed in such a way that when the ring's body undergoes some force deformation, the strain gauge resistance will also change. To measure the value of the octagonal ring strain and calculate the force, the data acquisition instrument will collect the signal using an electrical bridge circuit. Fig. 2a illustrates the shape of a single octagonal ring with eight strain gauges used to measure force in two directions. The resistance strain gauges No. 1 to No. 4 can measure the force in the $z$-axis direction $\left(F_{z}\right)$ as shown in Fig. 2b, while strain gauges No. 5 to No. 8 can measure the vertical force $\left(F_{x}\right)$ as shown in Fig. 2c. The friction stir welding dynamometer is composed of four octagonal rings, staggered around each other, to measure the force in three directions, as is shown in Fig. 3.

A drawing of the dynamometer is shown in Fig. 4.

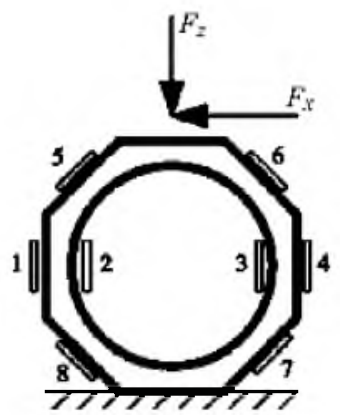

a

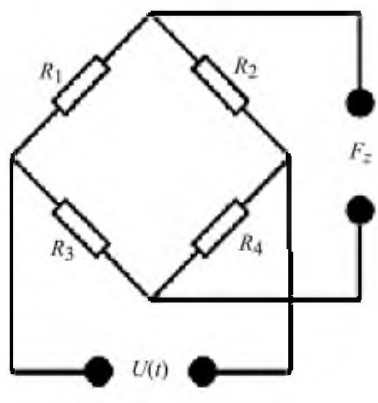

b

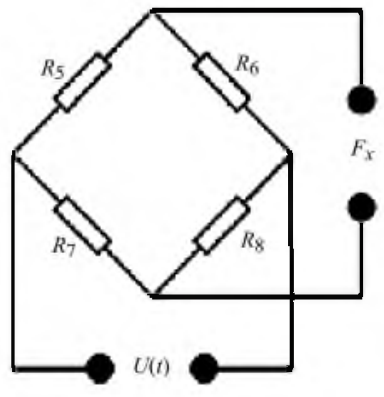

c

Fig. 2. Single octagonal ring measuring force principle [4]: (a) a single octagonal ring; (b) electric bridge circuit to measure the horizontal force; (c) electric bridge circuit to measure the vertical force. 


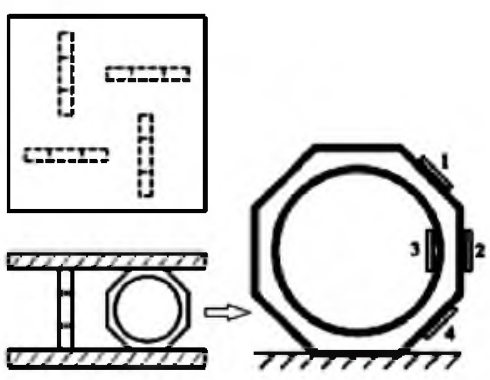

Fig. 3 Dynamometer gauge placement schematic.
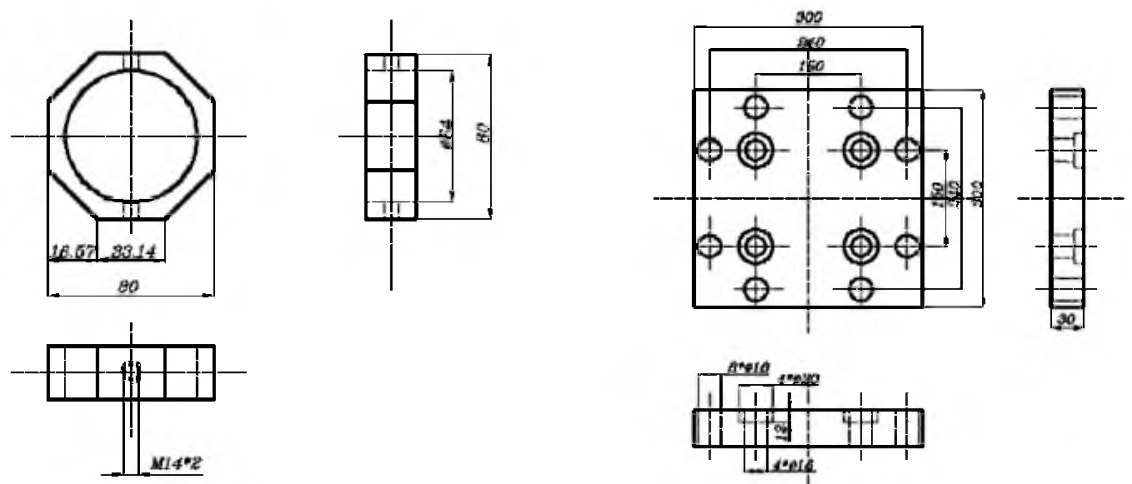

a

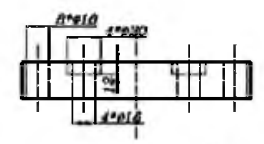

b
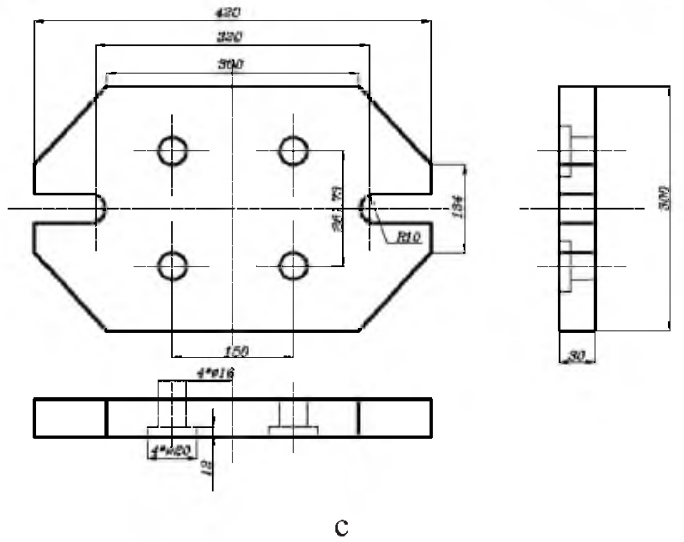

Fig. 4. The dynamometer drawing of friction stir welding: (a) octagonal ring drawing; (b) workbench drawing: (c) base drawing.

2. The Measuring Force Device and Its Calibration. The dynamometer dimensions are determined according to the three-directional force required and the aluminum alloy plate size needed for the friction stir welding force measurement experiment. Before manufacturing the dynamometer, the octagonal ring size, precision, roughness, and position are determined according to the sensitivity requirements. The dynamometer is divided in three parts: base, single octagonal ring, and workbench. The parts are connected by eight M $8 \times 50$ bolts. When the force is measured, the dynamometer connects a TST5915 dynamic strain testing system to computers for data acquisition, as shown in Fig. 5. The pressure $\left(F_{z}\right), x$-axis $\left(F_{x}\right)$ and $y$-axis $\left(F_{y}\right)$ forees are calibrated by a pressure sensor on the dynamometer. 


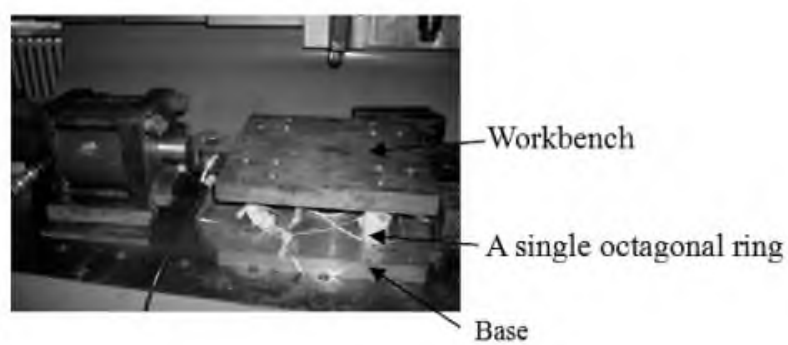

Fig. 5. Dynamometer calibration site photo.

The main principle behind the octagonal ring dynamometer's force measurement is that it indirectly measures the force by measuring the microstrain, caused by an external load, of the octagonal ring. Therefore, the dynamometer needs to be calibrated when running an actual force measurement. The force is measured by using a calibration value obtained from the measured microstrain. In this study, the $x$-, $y$-, and $z$-axis direction forces $\left(F_{x}, F_{y}\right.$, and $F_{z}$ ) are measured using the dynamometer. In the calibration process, the same directional forces are measured three times, and the average value is the final calibration value. The applied static load was $10,20,30,35,65,95$, or $130 \mathrm{~kg}$, respectively. The directional force calibration curves for the $x$-, $y$-, and $z$-axis directions are shown in Fig. 6. For an applied load in the $x$-axis direction, (Fig. 6a), it can be seen that the deformation in the $y$ - and $z$-axis directions was small enough to be neglected. For an applied load in the $y$-axis direction, (Fig. 6b), it is observed that deformation in the $x$ - and $z$-axis directions were small enough that it could be neglected. Similarly, for an applied load in the $z$-axis direction, (Fig. 6c), the deformation in the $x$ - and $y$-axis directions are small enough to be neglected. From linear tests, the calculated error percentages of $F_{x}, F_{y}$, and $F_{z}$ are $1.1,1.3$, and $1.2 \%$, respectively (Table 1 ). The three-directional cross sensitivity range is $0.4 \sim 2.1 \%$ (Table 2 ), which meets the dynamometer design requirements. Therefore, the $x$-, $y$-, and $z$-axis direction forces $\left(F_{x}, F_{y}\right.$, and $\left.F_{z}\right)$ are calibrated according to Fig. 6a, $b$, and $\mathrm{c}$, respectively.

$\mathrm{T}$ a b 1 e 1

The Linear Test Results of the Dynamometer

\begin{tabular}{|c|c|c|c|c|}
\hline Load direction & Load $(\mathrm{kg})$ & $\begin{array}{c}\text { Testing } \\
\text { microstrain } \mu \varepsilon\end{array}$ & $\begin{array}{c}\text { Calibration } \\
\text { microstrain } \mu \varepsilon\end{array}$ & Error (\%) \\
\hline$x$ & 50 & 30.3 & 30.7 & 1.1 \\
\hline$y$ & 50 & 10.4 & 10.3 & 1.3 \\
\hline$z$ & 50 & 40.9 & 41.4 & 1.2 \\
\hline
\end{tabular}

$\mathrm{T}$ a b 1 e 2

Dynamometer Cross Sensitivity Test Results

\begin{tabular}{|c|c|c|c|c|c|c|c|}
\hline \multirow{2}{*}{$\begin{array}{c}\text { Load } \\
\text { direction }\end{array}$} & \multirow{2}{*}{$\begin{array}{c}\text { Load } \\
(\mathrm{kg})\end{array}$} & \multicolumn{3}{|c|}{ Testing microstrain $\mu \varepsilon$} & \multicolumn{3}{c|}{ Average error (\%) } \\
\cline { 3 - 8 } & $X$ & $Y$ & $Z$ & $X$ & $Y$ & $Z$ \\
\hline$x$ & 50 & 30.3 & 0.13 & 0.15 & - & 0.4 & 0.5 \\
\hline$y$ & 50 & 0.04 & 10.4 & 0.22 & 0.4 & - & 2.1 \\
\hline$z$ & 50 & 0.73 & 0.31 & 40.9 & 1.8 & 0.8 & - \\
\hline
\end{tabular}




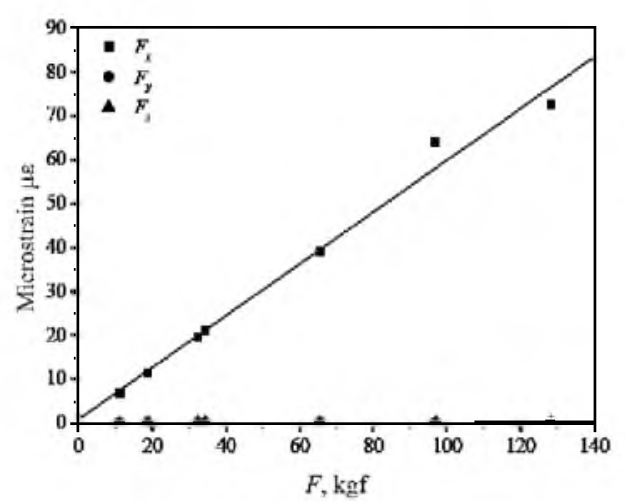

a

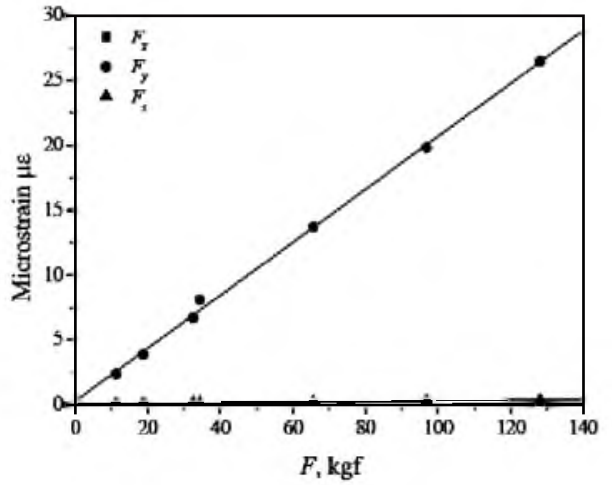

$\mathrm{b}$

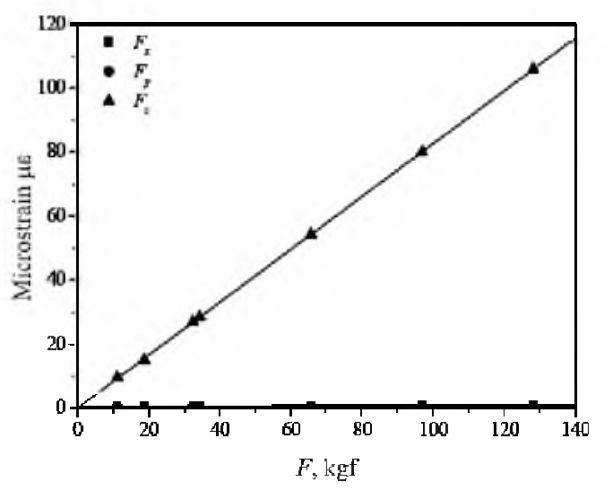

C

Fig. 6. Dynamometer calibration lines for: (a) $x$-axis direction, (b) $y$-axis direction, and (c) $z$-axis direction.

\section{Mathematical Model of the Stir Tool Force in a Friction Stir Welding Process.}

3.1. The Force Measurement Experiment. The four main process parameter for friction stir welding are: stir tool rotational speed $(\omega)$, welding speed $(v)$, under press value $(\Delta)$, and tilt angle $(\theta)$. Generally, in the welding process, due to the influence of the welding plate thickness and welding region quality, the plate thickness attained fixed $\Delta$ and $\theta$ values. In this work, four $10 \mathrm{~mm}$ thick 7022 aluminum alloy plates were used, keeping $\Delta=0.1 \mathrm{~mm}$ and $\theta=2.5^{\circ}$. The stir tool force direction used in the experiment is shown in Fig. 7. The results are shown in Table 3 .

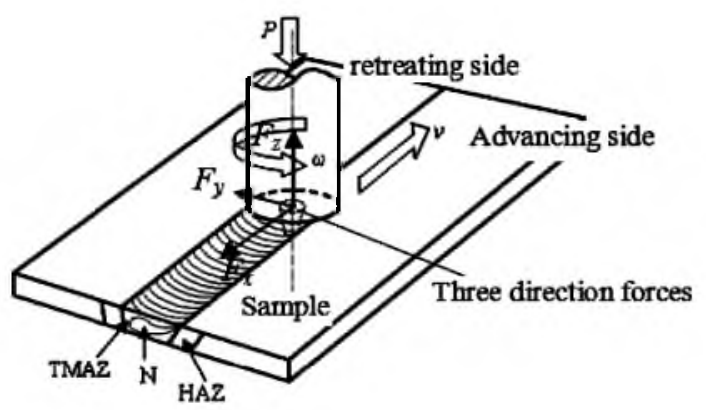

Fig. 7. The stir tool force direction. 
H. F. Wang, J. L. Wang, D. W Zuo, and W. W Song

$\mathrm{T}$ a b 1 e 3

Test Results of the Stir Tool Force Experiment

\begin{tabular}{|c|c|c|c|c|c|}
\hline Test No. & $\omega, \mathrm{rpm}$ & $v, \mathrm{~mm} / \mathrm{min}$ & $F_{x}, \mathrm{~N}$ & $F_{y}, \mathrm{~N}$ & $F_{z}, \mathrm{~N}$ \\
\hline 1 & 300 & 30 & 8387 & 1062 & 34650 \\
\hline 2 & 300 & 50 & 16060 & 1452 & 35680 \\
\hline 3 & 300 & 100 & 17190 & 2313 & 36130 \\
\hline 4 & 400 & 30 & 4130 & 905 & 28000 \\
\hline 5 & 400 & 50 & 7242 & 937.7 & 27680 \\
\hline 6 & 400 & 100 & 4401 & 1001 & 23860 \\
\hline 7 & 600 & 30 & 11980 & 953.4 & 24100 \\
\hline 8 & 600 & 50 & 14930 & 913.6 & 20750 \\
\hline 9 & 600 & 100 & 4187 & 580 & 9252 \\
\hline
\end{tabular}

3.2. Establishment of the Stir Tool Force Mathematical Model in the Friction Stir

Welding Process. There have not been any relevant reports that deal with a stir tool force mathematical model for friction stir welding processes. In this study, based on the experimental results and a regression analysis method, a stir tool force mathematical model for a friction stir welding process was established. Any function, at least in a small range, can be approximated by an arbitrary polynomial. Therefore, often in the more complex practical problems, regardless of the relationship between the dependent variable and each independent variable, a polynomial regression analysis is used.

Generally, in the field of science and technology, the quadratic polynomial approximation is accurate enough $[11,12]$. In this paper, the stir tool force mathematical model for the friction stir welding process of a $10 \mathrm{~mm}$ thick 7022 aluminum alloy was,

$$
\left\{\begin{array}{l}
F_{x}=a_{0}+a_{1} \omega+a_{2} v+a_{3} \omega v+a_{4} \omega^{2}+a_{5} v^{2} \\
F_{y}=b_{0}+b_{1} \omega+b_{2} v+b_{3} \omega v+b_{4} \omega^{2}+b_{5} v^{2} \\
F_{z}=c_{0}+c_{1} \omega+c_{2} v+c_{3} \omega v+c_{4} \omega^{2}+c_{5} v^{2}
\end{array}\right.
$$

The stir tool force mathematical model for the friction stir welding of a $10 \mathrm{~mm}$ thick 7022 aluminium alloy, Eq. (4), was obtained by substituting onto Eq. (3) the experimental results from Table 3 , and using regression analysis,

$$
\left\{\begin{array}{l}
F_{x}=49280-300.9 \omega+917.2 v-0.766 \omega v+0.372 \omega^{2}-4.455 v^{2} \\
F_{y}=4457-19.13 \omega+36.42 v-0.067 \omega v+0.023 \omega^{2}-0.029 v^{2} \\
F_{z}=65110-1616 \omega+368.9 v-0.766 \omega v+0.165 \omega^{2}-0.926 v^{2}
\end{array}\right.
$$

To test the regression effect of Eq. (4), two aspects of Eq. (4) should be analyzed: one is the overall effectiveness of the regression equation, and the second is the effect of each independent variable on the effectiveness of the regression.

First of all, Eq. (5) is used to solve for the RSS and ESS values,

$$
T S S=\Sigma\left(Y_{i}-\hat{Y}_{i}\right)^{2}+\Sigma\left(\hat{Y}_{i}-\bar{Y}\right)^{2}=R S S+E S S,
$$


where TSS is the total sum of deviation square, $R S S=\Sigma\left(Y_{i}-\hat{Y}_{i}\right)^{2}$ is the residual sum of squares, and $E S S=\Sigma\left(\hat{Y}_{i}-\bar{Y}_{i}\right)^{2}$ is the regression sum of squares.

Because $E S S$ is the regression sum of squares of the five independent variables, it has 5 degrees of freedom, while the RSS degrees of freedom is calculated using $(n-1)-5=$ $=n-6$. In this test, $n=9$, so RSS has 3 degrees of freedom.

Using mathematical statistics formulas, the $F$-test value is obtained using the following calculation:

$$
F=\frac{E S S / 5}{R S S / 3} .
$$

The $F_{\alpha,(5,3)}$ value is obtained from a look-up table, and then the validity of the regression equation can be assessed. Table 4.

According to Eqs. (5) and (6), the relevant variance analysis of Eq. (4) is listed in

$\mathrm{T}$ a b 1 e 4

Analysis of Variance

\begin{tabular}{|c|c|c|c|c|c|c|c|}
\hline Eq. & $\begin{array}{c}\text { Source } \\
\text { of variance }\end{array}$ & $\begin{array}{l}\text { The sum } \\
\text { of deviation } \\
\text { square }\end{array}$ & $\begin{array}{l}\text { Degree of } \\
\text { freedom }\end{array}$ & Variance & $F$ & $F_{01,(5,3)}$ & Significant \\
\hline \multirow[t]{3}{*}{$F_{x}$} & ESS & $2.23 \cdot 10^{8}$ & 5 & $4.46 \cdot 10^{7}$ & \multirow[t]{3}{*}{34.30} & \multirow[t]{3}{*}{5.31} & \multirow[t]{3}{*}{$*$} \\
\hline & RSS & $4.00 \cdot 10^{6}$ & 3 & $1.30 \cdot 10^{6}$ & & & \\
\hline & TSS & $2.27 \cdot 10^{8}$ & 8 & & & & \\
\hline \multirow[t]{3}{*}{$F_{y}$} & ESS & $1.69 \cdot 10^{6}$ & 5 & $3.38 \cdot 10^{5}$ & \multirow[t]{3}{*}{6.26} & \multirow[t]{3}{*}{5.31} & \multirow[t]{3}{*}{$*$} \\
\hline & RSS & $1.62 \cdot 10^{5}$ & 3 & $5.40 \cdot 10^{4}$ & & & \\
\hline & TSS & $1.85 \cdot 10^{6}$ & 6 & & & & \\
\hline \multirow[t]{3}{*}{$F_{z}$} & ESS & $6.03 \cdot 10^{8}$ & 5 & $1.21 \cdot 10^{8}$ & \multirow[t]{3}{*}{1480} & \multirow[t]{3}{*}{5.31} & \multirow[t]{3}{*}{$*$} \\
\hline & RSS & $2.45 \cdot 10^{5}$ & 3 & $8.17 \cdot 10^{4}$ & & & \\
\hline & TSS & $6.03 \cdot 10^{8}$ & 8 & & & & \\
\hline
\end{tabular}

In Eq. (4), for the analysis of linear regression equation, $\alpha=0.1$. In Table 4, three force linear equations were established under a $90 \%$ significance level.

An effectiveness analysis is performed for each independent variable with a $t$-test, with the variable $t_{i}$ being the calculated value for each independent variable.

The $t$-test quation is

$$
t_{i}=\frac{\sqrt{p_{i}}}{\sqrt{R S S / f}},
$$

where $f$ is the RSS degrees of freedom $(f=3)$ and $p_{i}$ is the partial regression sum of squares. In a linear regression, $p_{i}$ is an independent variable of the partial regression sum of squares that is used to get rid of the independent variables in the regression equation, and to reduce the regression sum of squares of the values. In other words, it does not contain the independent variable of the regression equation. When independent variables are added, 
the regression sum of squares increases. The $p_{i}$ term reflects the importance of the independent variables in the regression equation. Each variable of the partial regression sum of squares and $t$ values is listed in Table 5 .

From Table 5, it can be seen that for each of the three regression equations, one for each of the direction forces, the independent variables had $t>1$, except the $F_{y}$ equation, which had $t_{v}<1$. For the $F_{x}$ equation, each independent variable's impact on $F_{x}$ should not be neglected, with $\omega^{2}$ being more important on $F_{x}$ than other independent variables because it had a greater effect on $F_{x}$ than the other variables, while $v^{2}$ had the least impact. Although $t_{v}<1$ for $F_{y}$ shows that the $v$ variable had the least impact on $F_{y}$, it cannot be ignored, as it was observed that the value of $\omega v$ is more important for $F_{y}$ than all the other independent variables. In the $F_{z}$ equation, the influence of each independent variable on $F_{z}$ cannot be ignored, with $v^{2}$ having the strongest effect, while $\omega$ and $v$ have the lowest $t$ values.

$\mathrm{T}$ a b 1 e 5

The Independent Variable of the Partial Regression Sum of Squares and $t$ Values

\begin{tabular}{|c|c|c|c|c|c|}
\hline Eq. & $p_{\omega}$ & $p_{v}$ & $p_{\omega \nu}$ & $p_{\omega^{2}}$ & $p_{v^{2}}$ \\
\hline$F_{x}$ & $8.1 \cdot 10^{7}$ & $7.6 \cdot 10^{7}$ & $7.2 \cdot 10^{7}$ & $1.03 \cdot 10^{8}$ & $3.80 \cdot 10^{7}$ \\
\hline$F_{y}$ & $9.0 \cdot 10^{4}$ & $5.0 \cdot 10^{4}$ & $5.3 \cdot 10^{5}$ & $1.20 \cdot 10^{5}$ & $1.00 \cdot 10^{5}$ \\
\hline$F_{z}$ & $2.7 \cdot 10^{7}$ & $2.7 \cdot 10^{7}$ & $9.0 \cdot 10^{7}$ & $2.20 \cdot 10^{7}$ & $3.06 \cdot 10^{8}$ \\
\hline Eq. & $t_{\omega}$ & $t_{v}$ & $t_{\omega \nu}$ & $t_{\omega^{2}}$ & $t_{v^{2}}$ \\
\hline$F_{x}$ & 7.89 & 7.650 & 7.44 & 8.90 & 5.41 \\
\hline$F_{y}$ & 1.29 & 0.964 & 3.14 & 1.49 & 1.36 \\
\hline$F_{z}$ & 18.00 & 18.000 & 33.20 & 16.40 & 61.20 \\
\hline
\end{tabular}

In order to validate the correctness of the model, the model-based predictions and experimental values are listed in Table 6, where it can be seen that the stir tool force average error in the $x$-, $y$-, and $z$-axis directions was $10.4,4.66$, and $7.11 \%$, respectively. This implies that the values predicted by the established model coincide well with the experimental values, demonstrating the model accuracy. These results grant the model feasibility for subsequent friction stir welding finite element analysis.

T a b 1 e 6

Comparison of Model Predictions and Experimental Values

\begin{tabular}{|c|c|c|c|c|c|c|c|c|c|c|c|}
\hline \multirow[t]{2}{*}{ No. } & \multicolumn{2}{|c|}{$\begin{array}{l}\text { The process } \\
\text { parameters }\end{array}$} & \multicolumn{3}{|c|}{$F_{x}, \mathrm{~N}$} & \multicolumn{3}{|c|}{$F_{y}, \mathrm{~N}$} & \multicolumn{3}{|c|}{$F_{z}, \mathrm{~N}$} \\
\hline & $\omega$ & $v$ & $F_{x}^{t h e o r}$ & $F_{x}^{\exp }$ & $\begin{array}{c}\text { Error } \\
(\%)\end{array}$ & $F_{y}^{\text {theor }}$ & $F_{y}^{e x p}$ & $\begin{array}{c}\text { Error } \\
(\%)\end{array}$ & $F_{z}^{t h e o r}$ & $F_{z}^{\exp }$ & $\begin{array}{l}\text { Error } \\
(\%)\end{array}$ \\
\hline 1 & 500 & 30 & 3847 & 3541 & 8.64 & 703.5 & 740.2 & 4.96 & 24300 & 21679 & 12.1 \\
\hline 2 & 500 & 40 & 6070 & 6828 & 11.1 & 712.4 & 752.3 & 5.30 & 23510 & 22176 & 6.02 \\
\hline 3 & 500 & 60 & 7844 & 7516 & 4.36 & 712.8 & 738.1 & 3.43 & 21380 & 19872 & 7.59 \\
\hline 4 & 500 & 80 & 6054 & 7345 & 17.6 & 690 & 725.8 & 4.93 & 18500 & 19021 & 2.74 \\
\hline \multicolumn{5}{|c|}{ Average error $(\%)$} & 10.4 & & & 4.66 & & & 7.11 \\
\hline
\end{tabular}




\section{Conclusions}

1. A dynamometer suitable for friction stir welding was designed and manufactured. The dynamometer was calibrated, and the calibration results show that the error percentages for $F_{x}, F_{y}$, and $F_{z}$ are $1.1,1.3$, and $1.2 \%$, respectively. The three-directional cross sensitivity range is $0.4 \sim 2.1 \%$.

2. A mathematical model for the stir tool force exerted in friction stir welding processes was established.

3. For each axis direction, the model error between the predicted and experimental values fall within $18 \%$, which suggests that the established stir tool force model is applicable for the available friction stir welding equipment and technique. The predicted stir tool force theoretical value is reliable and provides the guaranteed accuracy for the finite element analysis of friction stir welding.

Acknowledgments. This study is supported by the National Natural Science Foundation of China (Grantes Nos. 51175255 and 51305199); the Excellent young talents fund key project of the Anhui Higher Education Institutions of China (Grant No. 2013SQRL089ZD); and the Starting Foundation for Talents from Huangshan University of China (Grant No. 2013xkjq003).

1. N. H. Cook and E. G. Loewen, "Metal cutting measurements and their interpretation," Proc. Amer. Soc. Exp. Stress Anal., 13, No. 3, 57-62 (1956).

2. N. H. Cook and E. Rabinowicz, Physical Measurement and Analysis, Addison-Wesley, New York (1963).

3. E. G. Loewen, E. R. Marshall and M. C. Shaw, "Electric strain gauge tool dynamometers," Proc. Amer. Soc. Exp. Stress Anal., 8, No. 2, 1-16 (1951).

4. C. Zhang and W. J. Li, "Design of STM32-based dynamometer," J. Test Measur. Tech., 25, No. 6, 515-518 (2011).

5. Z. Huang and W. Zhao, "Theoretical calculation and experimental analyses of natural frequency for high frequency dynamometer," China Mech. Eng., 26, No. 1, 7-11 (2015).

6. L. Y. Xu, J. Liu, K. N. Zhou, and S. Li, "Application of self-made dynamometer in measuring three-dimensional cutting forces of wood," Forest Eng., 25, No. 4, 49-52 (2009).

7. S. Karabay, "Analysis of drill dynamometer with octagonal ring type transducers for monitoring of cutting forces in drilling and allied process," Mater. Design, 28, 673685 (2007).

8. Y. Chen, N. B. McLaughlin, and S. Tessier, "Double extended octagonal ring (DEOR) drawbar dynamometer," Soil Till. Res., 93, 462-471 (2007).

9. H. F. Wang, Study on Friction Stir Jointing Technology and Milling Deformation Analysis of the Jointed Plate for 7022 Aluminum Alloy, Nanjing, Nanjing University of Aeronautics and Astronautics (2011).

10. M. J. O’Dogherty, "The design of octagonal ring dynamometers," J. Agr. Eng. Res., 63, 9-18 (1996).

11. S. H. Zheng and F. H. Jiang, Experimental Design and Data Processing, China Building Industry Press, Beijing (2004).

12. Z. X. Liu, R. H. Huang, and A. M. Tian, Experimental Design and Data Processing, China Chemical Press, Beijing (2005). 